\title{
Extracorporeal Immunoglobulin Elimination for the Treatment of Severe Myasthenia Gravis
}

\author{
M. Blaha, ${ }^{1}$ J. Pit'ha, ${ }^{2}$ V. Blaha, ${ }^{3}$ M. Lanska, ${ }^{1}$ J. Maly, ${ }^{1}$ S. Filip, ${ }^{4}$ and H. Langrova ${ }^{5}$ \\ ${ }^{1}$ 2nd Department of Internal Medicine, Hematology, Faculty of Medicine and Teaching Hospital, Charles University, \\ 50005 Hradec Králové, Czech Republic \\ ${ }^{2}$ Department of Neurology, 1st Faculty of Medicine and General Teaching Hospital, Charles University, 12000 Prague, Czech Republic \\ ${ }^{3}$ Department of Metabolic Care and Gerontology, Faculty of Medicine and Teaching Hospital, Charles University, \\ 50005 Hradec Králové, Czech Republic \\ ${ }^{4}$ Department of Radiotherapy and Oncology, Faculty of Medicine and Teaching Hospital, Charles University, \\ 50005 Hradec Králové, Czech Republic \\ ${ }^{5}$ Department of Ophthalmology, Faculty of Medicine and Teaching Hospital, Charles University, 50005 Hradec Králové, Czech Republic
}

Correspondence should be addressed to M. Blaha, blaham@email.cz

Received 1 December 2009; Accepted 17 January 2010

Academic Editor: Guy M. Benian

Copyright ( $) 2010$ M. Blaha et al. This is an open access article distributed under the Creative Commons Attribution License, which permits unrestricted use, distribution, and reproduction in any medium, provided the original work is properly cited.

Myasthenia gravis (MG) is a neuromuscular disorder leading to fluctuating muscle weakness and fatigue. Rarely, long-term stabilization is not possible through the use of thymectomy or any known drug therapy. We present our experience with extracorporeal immunoglobulin (Ig) elimination by immunoadsorption (adsorbers with human Ig antibodies). Acetylcholine receptor antibodies (AChRAs) were measured during long-term monitoring (4.7 \pm 2.9 years; range 1.1-8.0). A total of 474 samples (232 pairs) were analyzed, and a drop in AChRA levels was observed $(P=.025)$. The clinical status of patients improved and stabilized. Roughly $6.8 \%$ of patients experienced clinically irrelevant side effects. The method of Ig elimination by extracorporeal immunoadsorption (IA) is a clinical application of the recent biotechnological advances. It offers an effective and safe therapy for severe MG even when the disease is resistant to standard therapy.

\section{Introduction}

Myasthenia gravis (MG) is an autoimmune disorder in which weakness is caused by circulating acetylcholine receptor antibodies (AchRAs) or muscle-specific kinase antibodies that block acetylcholine receptors at the postsynaptic neuromuscular junction [1]. MG is treated with cholinesterase inhibitors or immunosuppressive drugs. Surgical removal of the thymus (thymectomy) may result in permanent remission or a reduced need for drug therapy. When myasthenia is serious (myasthenic crisis), plasmapheresis can be used to remove putative antibodies from the circulation. Intravenous immunoglobulins (IVIGs) can also be used to bind the circulating antibodies [2-5]. Both of these treatments have relatively short-lived benefits, which are typically measured in weeks [6]. When some patients are unresponsive to such therapy while on the maximum-tolerated drug doses and sufficient stabilization is not achieved, they may be threatened by repeated myasthenic crises. Removal of the pathogenic agents from the blood may improve the patient's condition in these cases. Due to advances in biomedical technology, adsorbers with specific affinity for immunoglobulin $\mathrm{G}(\operatorname{IgG})$ and the pathogenic antiacetylcholine receptor antibody of MG [7] have been developed. In severe MG resistant to other treatments, extracorporeal elimination (EE) of immunoglobulins by immunoadsorption (IA) has been used as either a periodic cure [8] or long-term regular therapy.

In this paper, we review our use of EE of IgGs via adsorbers containing sheep antibodies against human IgG as a long-term therapy for severe MG refractory to standard drug therapy and thymectomy. 


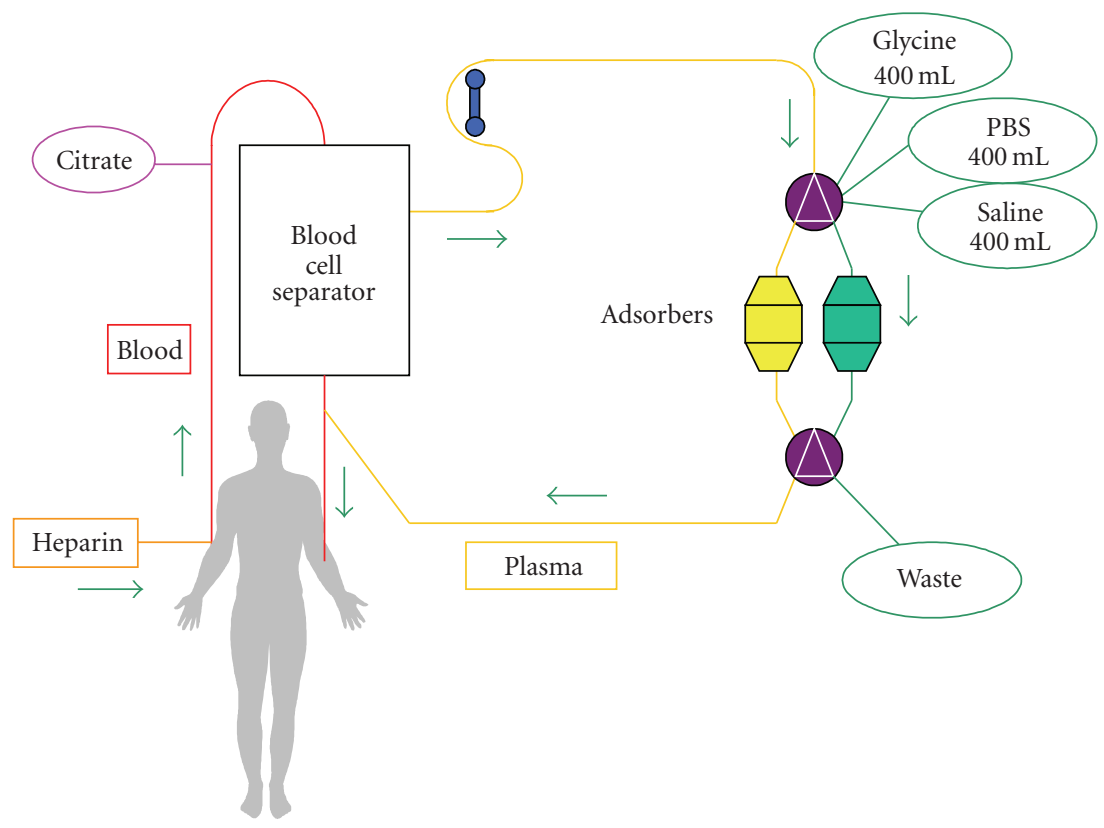

FIGURE 1: Our modification of immunoadsorption (modified by Borberg et al. [9, 10]). Vascular access for the extracorporeal circuit is established via two peripheral veins. Plasma without cellular elements obtained after high-speed centrifugation with a quality separator (Cobe-Spectra, Denver, USA) is drawn through an adsorbent Adsopak capsule. Plasma flow is continuous. After filling the adsorbent capsule, the Adasorb device (Medicap, Ulrichsteinn, Germany) switches automatically to the second adsorbent capsule; the first capsule is washed with glycine, PBS buffer, and saline to prepare it for reuse so the procedure can be repeated as necessary. Regeneration solutions are drained into a waste bag. Clean, washed plasma flows from the column back to the separator where it is mixed with blood elements and returns to the patient's peripheral veins. Anticoagulation is maintained by a continuous supply of citrate solution (ACD-A Baxter, Germany), and it is secured by an initial application of $2500 \mathrm{U}$ heparin i.v., subsequent continuous infusion of $50 \mathrm{U}$ of heparin/min and gradual dose lowering such that heparin application ceases in the middle of immunoadsorption.

\section{Patients and Methods}

2.1. Extracorporeal Elimination by Immunoadsorption. Here, we describe our own modification of an immunoadsorption method presented by Borberg et al. [9, 10]. The procedure consists of two main steps: (1) separation of plasma by continuous centrifugation in a Cobe-Spectra separator (Cobe, Denver, USA) and (2) subsequent passage of plasma through a pair of Adsopak 200 adsorbers (Pocard, Moscow, Russia) placed in an automatic adsorption-desorption device (Adasorb, Medicap, Ulrichstein, SRN). The device switches between two adsorbent capsules and finally returns plasma back to the patient. As soon as one of the adsorbent capsules is full, the automatic device switches to the second capsule; the first capsule is washed and prepared to be used again. The adsorption cycles are repeated according to the clinical condition of the patient up to ten times.

The Adsopak 200 adsorption column contains $200 \mathrm{~mL}$ of antihuman IgG Sepharose 4FF gel (binding capacity of 10-12 mg Ig protein/1 mL of adsorbent) and approximately $85 \mathrm{~mL}$ of phosphate buffer ( $\mathrm{pH} 7.4)$. Polyclonal antibodies against human Ig bound to Sepharose 4FF are prepared from sheep serum immunized by human IgG. Adsopak $200 \mathrm{Ig}$ columns are intended for use in therapeutic Ig apheresis procedures for patients requiring rapid reductions of immunoglobulin concentrations in the plasma. For the therapeutic scheme, see Figure 1.
2.2. Patients. This prospective observational study was conducted at the 2nd Department of Medicine, Hemapheretic Center of Charles University, Faculty of Medicine, Hradec Kralove, Czech Republic. In the Czech Republic, there are two main centers for the treatment of myasthenia gravis. Smaller centers are in other teaching hospitals. Presently, 1916 patients are treated at the Myasthenic Center in Prague. Only six of these patients were in severe condition despite thymectomy and drug therapy (including corticosteroids, immunosuppressive drugs and repeated doses of immunoglobulins) and could not be stabilized for a longer period of time. Therefore, these patients were included in the program of long-term therapy with extracorporeal elimination of immunoglobulins. Basic clinical data are given in Table 1.

All patients were women with a mean age of 46 years (range: 28-65 years). Patients 1 and 2 were successfully weaned off of EE therapy after 13 months and are on longterm immunosuppressive therapy and permanent care at the Myasthenic Center. Patients 3-6 are on long-term EE treatment (mean: 6.5 years, range: 5-8 years). Attempts to reduce therapeutic intensity by weaning off of EE were not successful in these patients. Patients 3 and 5 suffer from other diseases (hypertension, ischemic heart disease, and arthritic symptoms) compensated for by conventional therapy. Patients 5 and 6 experienced attacks of secondary depressive syndrome, and patient 4 attempted to commit 
TABle 1: Basic clinical data. Number of myasthenic crises experienced requiring artificial ventilation: $+=1-2,++=2-5,+++=$ more than 5. IA = immunotherapy, Mes: Mestinon, M: Medrol, P: Prednison, CC: CellCept, Pr: Prograf.

\begin{tabular}{lccccccc}
\hline Patient no. Classification (MGFA) & Age & Sex & Myasthen. crises & Therapy duration & Current condition & Current therapy \\
\hline 1 & III b & 35 & F & ++ & 13 & Excellent, IA therapy stopped & Mes \\
2 & IV b & 33 & F & + & 13 & Stabilization, IA therapy stopped & Mes \\
3 & IV b & 65 & F & +++ & 96 & Stabilization & Mes, M, CC, IVIG \\
4 & IV b & 28 & F & +++ & 76 & Stabilization & Mes, P, Pr \\
5 & III b & 53 & F & + & 79 & Stabilization & Mes, P, CC, IVIG \\
6 & IV b & 60 & F & ++ & 62 & Stabilization & Mes, P, CC \\
\hline
\end{tabular}

TABLe 2: AChRA levels. $N$ = number of patients. Normal AChRA values in our laboratory: 0.00-0.40 nmol/L.

\begin{tabular}{|c|c|c|c|c|c|c|c|c|}
\hline \multirow{2}{*}{ Patient no. } & \multicolumn{4}{|c|}{ AChRA before the procedures (ng/L) } & \multicolumn{4}{|c|}{ AChRA after the procedures (ng/L) } \\
\hline & $N$ & Mean \pm SD & Range & Median & $N$ & Mean \pm SD & Range & Median \\
\hline All & 232 & $12.8 \pm 11.4$ & $2.6-99.7$ & 11.2 & 231 & 5.46 & $1.3-57.3$ & 5.5 \\
\hline 1 & 13 & $51.4 \pm 20.9$ & $23.7-99.7$ & 49.6 & 13 & $28.0 \pm 12.8$ & $13.2-57.3$ & 23.3 \\
\hline 2 & 12 & $4.8 \pm 0.6$ & $4.0-6.0$ & 5.0 & 12 & $2.4 \pm 0.7$ & $1.6-4.0$ & 2.3 \\
\hline 3 & 43 & $5.5 \pm 1.2$ & $3.5-9.0$ & 5.4 & 43 & $2.3 \pm 0.5$ & $1.3-3.5$ & 2.4 \\
\hline 4 & 71 & $11.9 \pm 2.2$ & $7.9-19.0$ & 11.4 & 70 & $4.4 \pm 0.8$ & $2.8-7.1$ & 4.3 \\
\hline 5 & 58 & $15.3 \pm 3.0$ & $11.2-26.0$ & 15.1 & 58 & $6.3 \pm 1.0$ & $4.8-9.0$ & 6.3 \\
\hline 6 & 35 & $7.8 \pm 2.0$ & $2.6-14.4$ & 7.6 & 35 & $2.7 \pm 0.4$ & $2.2-4.4$ & 2.6 \\
\hline
\end{tabular}

suicide when in personal crisis. All patients are AChRApositive. All underwent thymectomy at the beginning of therapy with subsequent irradiation. A thymoma was found in patient 6.

2.3. Ig and AChRA Examination. The levels of IgG, IgA, and IgM were determined using the immunonephelometric method (IMMAGE 800 System, Beckman Coulter, USA). Determination of autoantibodies against the acetylcholine receptor was carried out by radio receptor assay for the in vitro diagnostic quantitative determination of autoantibodies against the acetylcholine receptor in human serum and plasma (IBL International, Hamburg, Germany).

2.4. Statistics. Statistical calculations were performed with SYSTAT 8 software. A standard paired $t$-test (Paired Comparison $t$-Test) was used to evaluate changes before and after the procedures. The dependence of AChRA values on the number of procedures was calculated by linear regression (Simple Linear Regression). Differences were considered statistically significant when $P \leq .05$.

\section{Results}

3.1. AChRA Levels. To investigate AChRA levels, a total of 464 samples were collected (232 paired examinations). Because one measurement was defective, levels in 463 samples were measured. The results are presented in Table 2. The first line summarizes the results for all patients, and the remaining six lines present evaluations of individual patients. After the procedures, the drop in AChRA levels was 57\% on

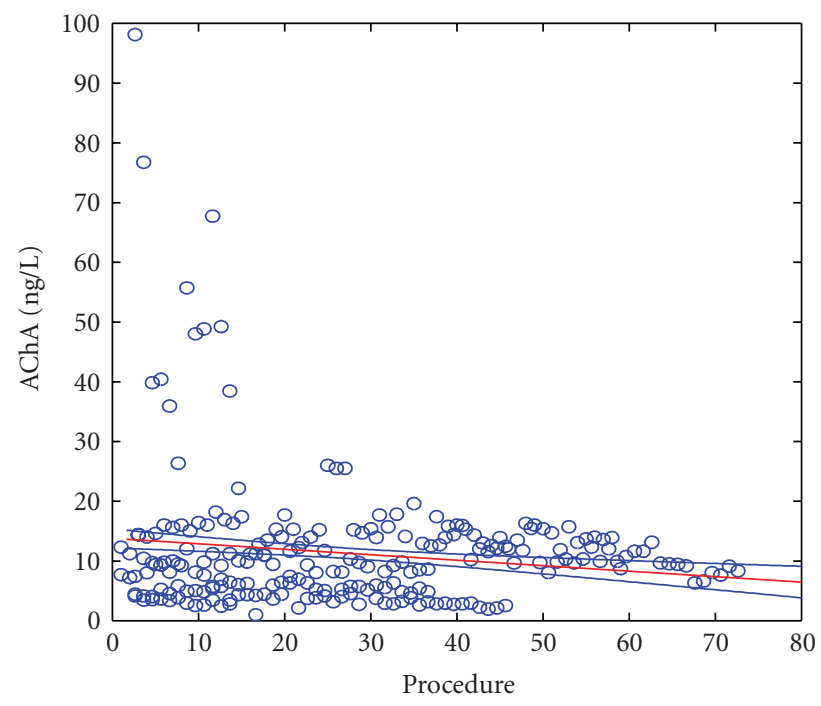

FIgure 2: AChRA levels-all examinations. $Y$ axis = AChRA level in $\mathrm{ng} / \mathrm{L} ; X$ axis: Procedure $=$ numeral order of procedures from the start of therapy.

an average; a paired $t$-test revealed a significance value of $P<.0001$.

Linear correlation analysis showed that a gradual decrease in AChRA levels occurred after repeated procedures $(P=.025)$ (Figure 2).

However, an evaluation of individual patients by linear regression showed that an important drop of AChRA levels $(P \leq .05)$ was found only in patients 1,2 , and 3 . In other 


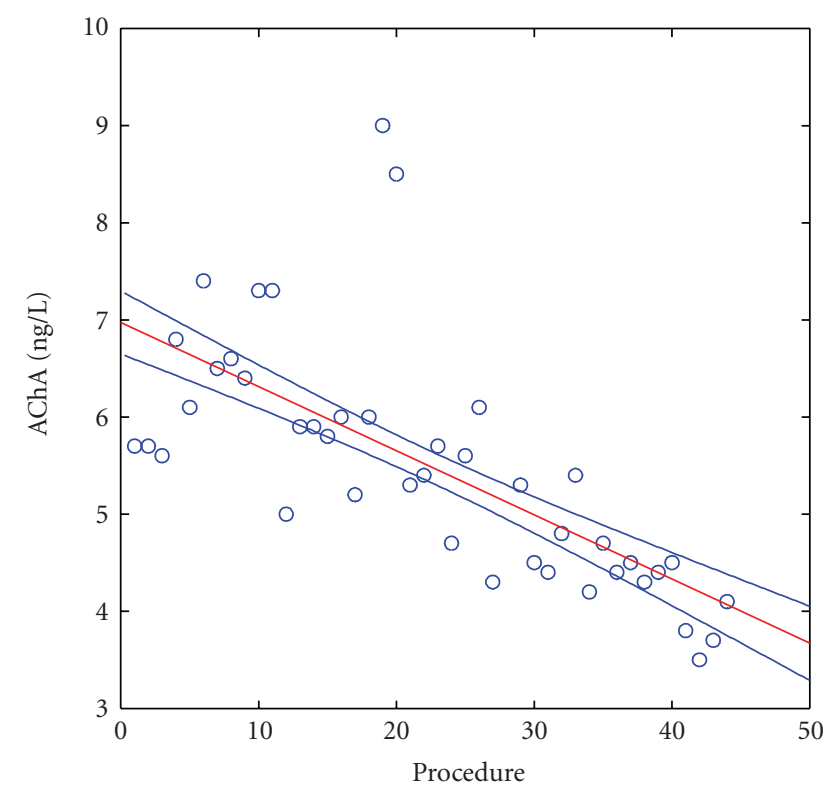

Figure 3: AChRA levels in patient no. 3. $X$ axis: Procedure $=$ numeral order of procedures from the start of therapy; $Y$ axis = AChRA levels in $\mathrm{ng} / \mathrm{L}$. Normal value in our laboratory $=0.00$ $0.40 \mathrm{nmol} / \mathrm{L}$.

patients, AChRA levels did not significantly change. As a positive example $(P \leq .0001)$, we present patient 3 (see Figure 3). In this patient, a gradual decrease in AChRA levels occurred during long-term therapy (i.e., a level of $7.1 \mathrm{ng} / \mathrm{L}$ was measured before the first IA, and the last measurement three years later (September 5, 2009) was $4.1 \mathrm{ng} / \mathrm{L}$ ). During these three years, the patient's condition was relatively stable without myasthenic crisis requiring artificial ventilation. (Figures 2 and 3, $X$ axis: Procedures $=$ numerical order of procedures from the start of therapy.)

In the rest of the patients, no gradual drop in AChRA levels was observed. Levels at the start of the therapy did not differ in a statistically significant manner from either those further in the course of illness or the last three values.

3.2. Immunoglobulins. Ig levels were examined in all patients regularly at the same time as AChRA levels. Table 3 shows that the levels of all three Ig classes significantly decreased after the procedures. This was most obvious for $\operatorname{IgG}(53.9 \%$ decrease) but was also true for IgM (42.5\% decrease) and IgA (44.7\% decrease). Correlation analysis did not find any dependence between the drop in Ig levels and AChRA levels.

3.3. Adverse Effects. IA is a kind of extracorporeal circulation that brings certain risks. However, the modifications presented here allowed it to be a safe and technically feasible method. Only $6.8 \%$ of patients in the 232 procedures experienced side effects, and none of these were life-threatening. Patient 3 once experienced aspiration of excreta during a cough attack and was treated with a short observation and bed rest. Vasovagal events (symptoms of neurovegetative lability) were the most common adverse effects manifested as malaise, weakness, slight and shortterm drop of blood pressure, or other general signs. All were well controlled by symptomatic therapy (Trendelenburg's position, cessation of the procedure and eventual quick dropping of saline). These reactions were under control in some tens of seconds or a few minutes at maximum. An ECG showed sinus bradycardia under a level of 50/min (46 and $48 / \mathrm{min}$ ) in two cases. This event occurred in patient 6 , but observation and short bed rest provided sufficient treatment.

\section{Discussion}

Myasthenia gravis is a rather rare neuromuscular disease of autoimmune origin. However, its course can be severe or even life-threatening. The annual incidence is from 2 to 4 million cases [11]. Most MG patients can be stabilized for a long time with either thymectomy or the application of symptomatic drugs, corticoids, immunosuppressives, immunoglobulins, or monoclonal agents. Myasthenic crises are mostly resolved by plasma exchange. This therapy is used in the few patients that are difficult to stabilize by any kind of drug therapy. Advances in biotechnology bring new knowledge and new methods that can be used in many branches of medicine [12-16]. One such advance is extracorporeal therapy, which can be either nonspecific (such as plasma exchange) or specific (such as IA). This type of procedure can constitute an important and sometimes decisive component of MG therapy. IA with regard to medical, technical, and economic demands is indicated in cases when classical methods fail. We attempted to manage this situation by extracorporeal elimination of AChRAs present in the IgG fraction. This was possible by the development of adsorbers that retain the clinically significant Ig component from extracorporeal plasma circulation. Adsopak adsorbers were used. These adsorbers contain antihuman IgG antibodies with a binding capacity of about $10 \mathrm{mg} \mathrm{Ig}$ protein per $1 \mathrm{~mL}$ of adsorbent. This capacity was sufficient to reduce AChRA levels by an average of $58 \%$ after the procedures.

The patients had different initial AChRA levels that did not correlate with the clinical activity of the disease [17] (Table 2). In all patients, AChRA levels significantly decreased (by about $57.7 \%$ on average) after the procedures $(P<.0001)$. With regard to the clinical effect of therapy, the best result was observed in patient 1. This patient is now maintained on a small dose of pyridostigmin. She has born a healthy child and enjoys a happy family life without physical limitations. Presently, she does not experience any muscular weakness. Similarly, patient 2 was weaned off the IA program after 13 months after stabilization and clinical improvement. This patient is now on symptomatic medication but experiences residual weakness of the oropharyngeal muscles of small functional significance. Patients 3-6 are still under a periodic EE regime. Attempts to wean them off of treatment were not successful. In patient 4 , procedures are repeated at 4-week intervals. In patients 4 and 5 , the procedures are performed 
TABLe 3: Immunoglobulins. $N$ : number of patients, SD: standard deviation.

\begin{tabular}{lccccccccccc}
\hline \multirow{2}{*}{ Level $(\mathrm{g} / \mathrm{L})$} & \multicolumn{4}{c}{ Before immunoapheresis } & \multicolumn{9}{c}{ After immunoapheresis } \\
& $N$ & Average & SD & Range & Median & $N$ & Average & SD & Range & Median & $P$ \\
\hline IgG & 240 & 7.93788 & 2.41842 & $1.59-14.2$ & 7.25 & 239 & 3.65904 & 1.80996 & $1.51-12.3$ & 3.21 & .000000 \\
IgM & 240 & 0.94921 & 0.32584 & $0.18-2.01$ & 0.955 & 239 & 0.54782 & 0.2622 & $0.15-1.51$ & 0.54 & .000000 \\
IgA & 240 & 2.32754 & 1.02688 & $0.24-4.51$ & 2.44 & 239 & 1.28661 & 0.70743 & $0.23-4.07$ & 1.22 & .000000 \\
\hline
\end{tabular}

at 3-week intervals. Patient 4 attempted to change to 4 -week intervals after 23 months of EE but shortly thereafter had to return to 3 -week intervals again. In patient 5, the IA program was initiated at 3-week intervals; after six months, 4-week intervals became sufficient. Patients 3 and 5 are on a periodic regime of EE followed by an IVIG dose performed during their last year of therapy. This therapy is sufficient for long-term stabilization. However, the clinical condition of patient 5 worsened during an attempt to wean off IVIG that led to a significant increase of AChAR levels; therefore, the IA regime has been continued.

It is well known that MG is more frequent in younger women and older men $[18,19]$. Our group comprised only women. Due to the small number of patients in our group, this bias was due to mere chance.

We monitored the basic parameters of clinical status (blood pressure, pulse and breath frequency) in all of our patients. As IA is one method of extracorporeal circulation, all basic hematologic and biochemical parameters (blood count, minerals, creatinin, glycemia and total protein, albumin, bilirubin, aminotransferase, and lipoprotein levels; data not shown) were regularly monitored. The results showed that clinically significant changes did not occur.

The drop in Ig after immunoaphereses was significant, but Ig levels returned to their initial values in the time before the next procedure. The drop in Ig levels might lead to increased susceptibility to infections, but we did not observe such susceptibility. Furthermore, we did not find in the literature data or reports describing higher infection rates after immunoapheresis for MG [18] or immunoaphereses performed for other indications (e.g., LDL-apheresis in familial hypercholesterolemia) [9].

\section{Conclusion}

The IA method presented here utilizes current results and recent advances in biotechnology and is indicated in severe cases of MG when longer-term stabilization cannot be achieved by another means. The levels of AChRA are significantly lower after the procedures. Reduction of AChRA levels can also be observed when patients are evaluated chronologically (i.e., the levels were higher at the beginning of follow-up than at the end of monitoring). This method is medically, technically, and economically demanding. However, it offers a clinically effective method by which to improve or stabilize patients with severe MG, even when the disease is resistant to all standard therapies. In the long run, this method protects patients against myasthenic crises, and it is safe with a low occurrence of side-effects.

\section{Acknowledgment}

The work was supported by a grant from the Internal Grant Agency, Ministry of Health, Czech Republic, no. NS/9743-4.

\section{References}

[1] B. M. Conti-Fine, M. Milani, and H. J. Kaminski, "Myasthenia gravis: past, present, and future," Journal of Clinical Investigation, vol. 116, no. 11, pp. 2843-2854, 2006.

[2] J. Díaz-Manera, R. Rojas-García, and I. Illa, "Treatment strategies for myasthenia gravis," Expert Opinion on Pharmacotherapy, vol. 10, no. 8, pp. 1329-1342, 2009.

[3] R. Gold and C. Schneider-Gold, "Current and future standards in treatment of myasthenia gravis," Neurotherapeutics, vol. 5, no. 4, pp. 535-541, 2008.

[4] R. Manfredi, G. Fasulo, C. Fulgaro, and S. Sabbatani, "Associated thyreoiditis, myasthenia gravis, thymectomy, Chron's disease, and erythema nodosum: pathogenetic and clinical correlations, immune system involvement, and systemic infectious complications," Rheumatology International, vol. 28, no. 11, pp. 1173-1175, 2008.

[5] H. Borberg, C. Jimenez, M. Belák, W. F. Haupt, and P. Späth, "Treatment of autoimmune disease by immunomodulation through extracorporeal elimination and intravenous immunoglobulin," Transfusion Science, vol. 15, no. 4, pp. 409418, 1994.

[6] C. J. Symonette, B. V. Watson, W. J. Koopman, M. W. Nicolle, and T. J. Doherty, "Muscle strength and fatigue in patients with generalized myasthenia gravis," Muscle \& Nerve, vol. 41, no. 3, pp. 362-9, 2010.

[7] S. Nakaji and N. Hayashi, "Adsorption column for myasthenia gravis treatment: medisorba MG-50," Therapeutic Apheresis and Dialysis, vol. 7, no. 1, pp. 78-84, 2003.

[8] N. I. Triantafyllou, E. I. Grapsa, E. Kararizou, E. Psimenou, A. Lagguranis, and A. Dimopoulos, "Periodic therapeutic plasma exchange in patients with moderate to severe chronic myasthenia gravis non-responsive to immunosuppressive agents: an eight year follow-up," Therapeutic Apheresis and Dialysis, vol. 13, no. 3, pp. 174-178, 2009.

[9] H. Borberg, "28 years of LDL-apheresis: a review of experience," Transfusion and Apheresis Science, vol. 41, no. 1, pp. 49-59, 2009.

[10] H. Borberg, R. Brunner, M. Tauchert, and R. Widder, "The current state of extracorporeal haemorheotherapy: from haemodilution via cascadefiltration to rheohaemapheresis," Transfusion and Apheresis Science, vol. 24, no. 1, pp. 57-64, 2001.

[11] F. Romi, N. E. Gilhus, and J. A. Aarli, "Myasthenia gravis: clinical, immunological, and therapeutic advances," Acta Neurologica Scandinavica, vol. 111, no. 2, pp. 134-141, 2005. 
[12] R. D. Sleator and C. Hill, "Rational design of improved pharmabiotics," Journal of Biomedicine and Biotechnology, vol. 2009, Article ID 275287, 7 pages, 2009.

[13] L.-H. Zhang, L. Y. Wu, S. Zhang, et al., "Anti-hepatitis B virus $\mathrm{X}$ protein in sera is one of the markers of development of liver cirrhosis and liver cancer mediated by HBV," Journal of Biomedicine and Biotechnology, vol. 2009, Article ID 289068, 6 pages, 2009.

[14] S. Karray-Chouayekh, F. Trifa, and A. Khabir, "Clinical significance of epigenetic inactivation of hMLH1 and BRCA1 in tunisian patients with invasive breast carcinoma," Journal of Biomedicine and Biotechnology, vol. 2009, Article ID 369129, 7 pages, 2009.

[15] M. Iga, J. H. P. Robertson, M. C. Winslet, and A. M. Seifalian, "Clinical potential of quantum dots," Journal of Biomedicine and Biotechnology, vol. 2007, Article ID 76087, 10 pages, 2007.

[16] A. El Andaloussi and C. Bilhou-Nabera, "New complex chromosomal translocation in chronic myeloid leukaemia: $\mathrm{T}(9 ; 18 ; 22)$ (q34;p11;q11)," Journal of Biomedicine and Biotechnology, vol. 2007, Article ID 92385, 3 pages, 2007.

[17] M. N. Meriggioli and D. B. Sanders, "Autoimmune myasthenia gravis: emerging clinical and biological heterogeneity," The Lancet Neurology, vol. 8, no. 5, pp. 475-490, 2009.

[18] J. F. Howard Jr., Myasthenia Gravis: A Manual for the Health Care Provider, Myasthenia Gravis Foundation of America, St. Paul, Minn, USA, 2008.

[19] L. M. Chiang, B. T. Darras, and P. B. Kang, "Juvenile myasthenia gravis," Muscle \& Nerve, vol. 39, no. 4, pp. 423$431,2009$. 

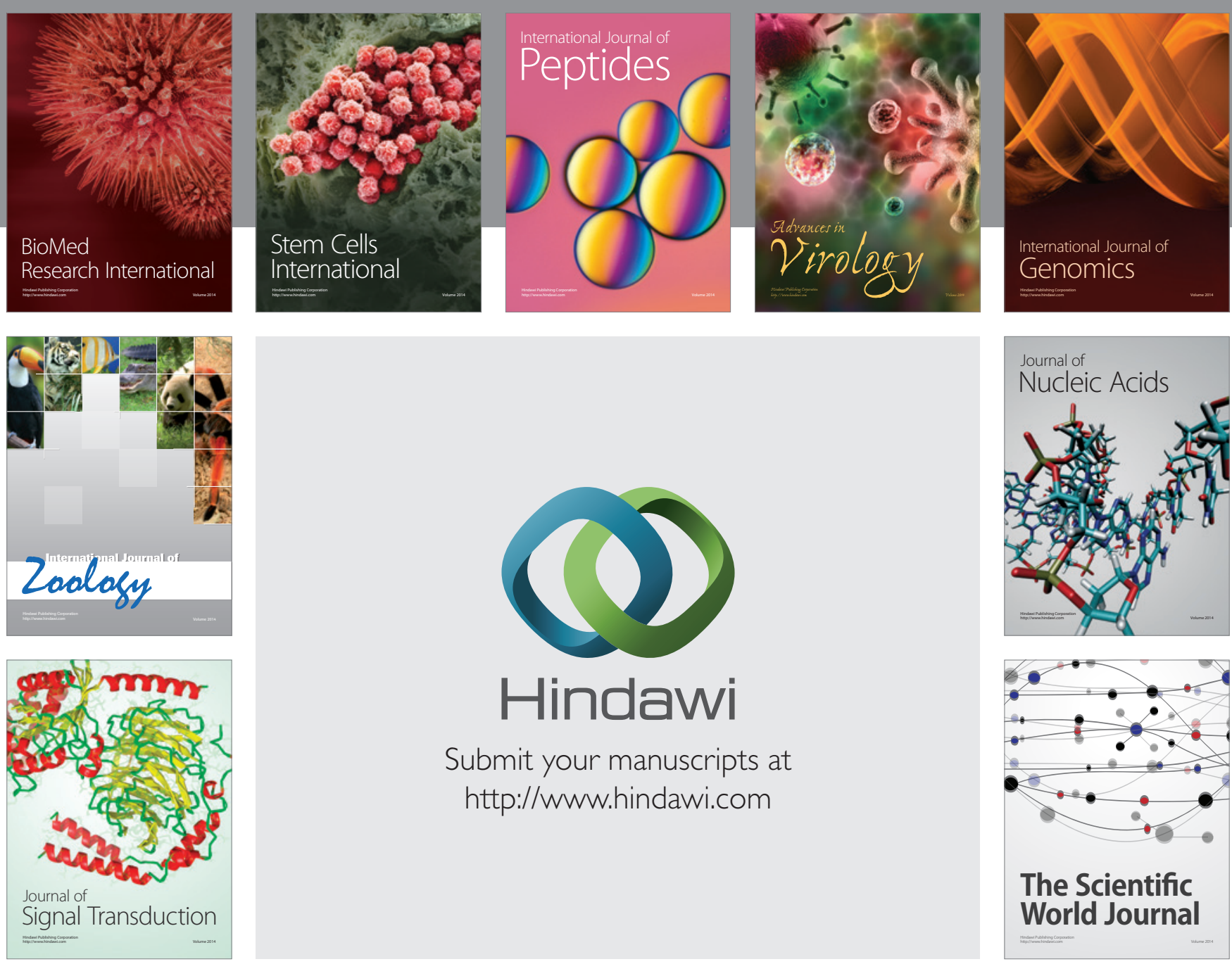

Submit your manuscripts at

http://www.hindawi.com
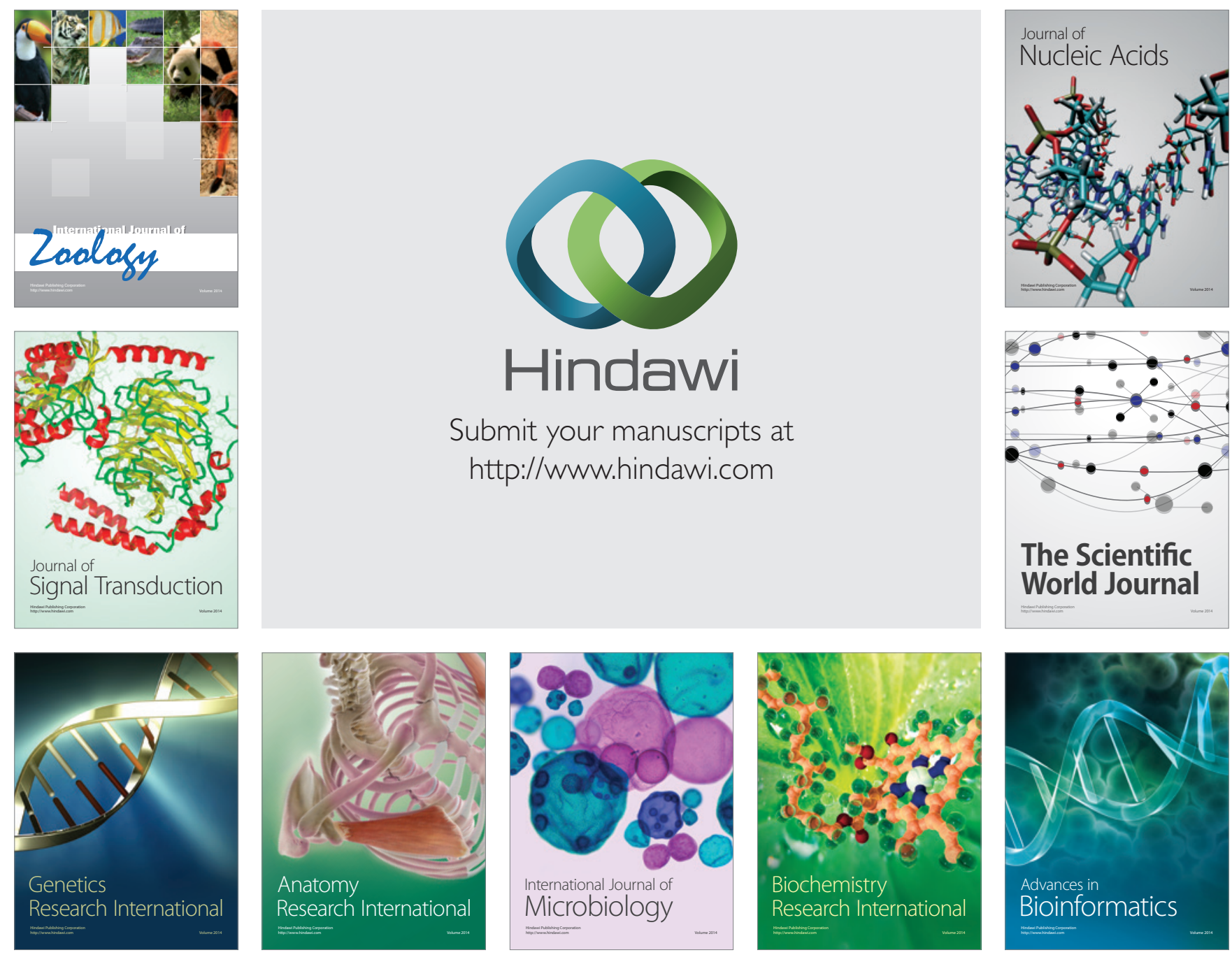

The Scientific World Journal
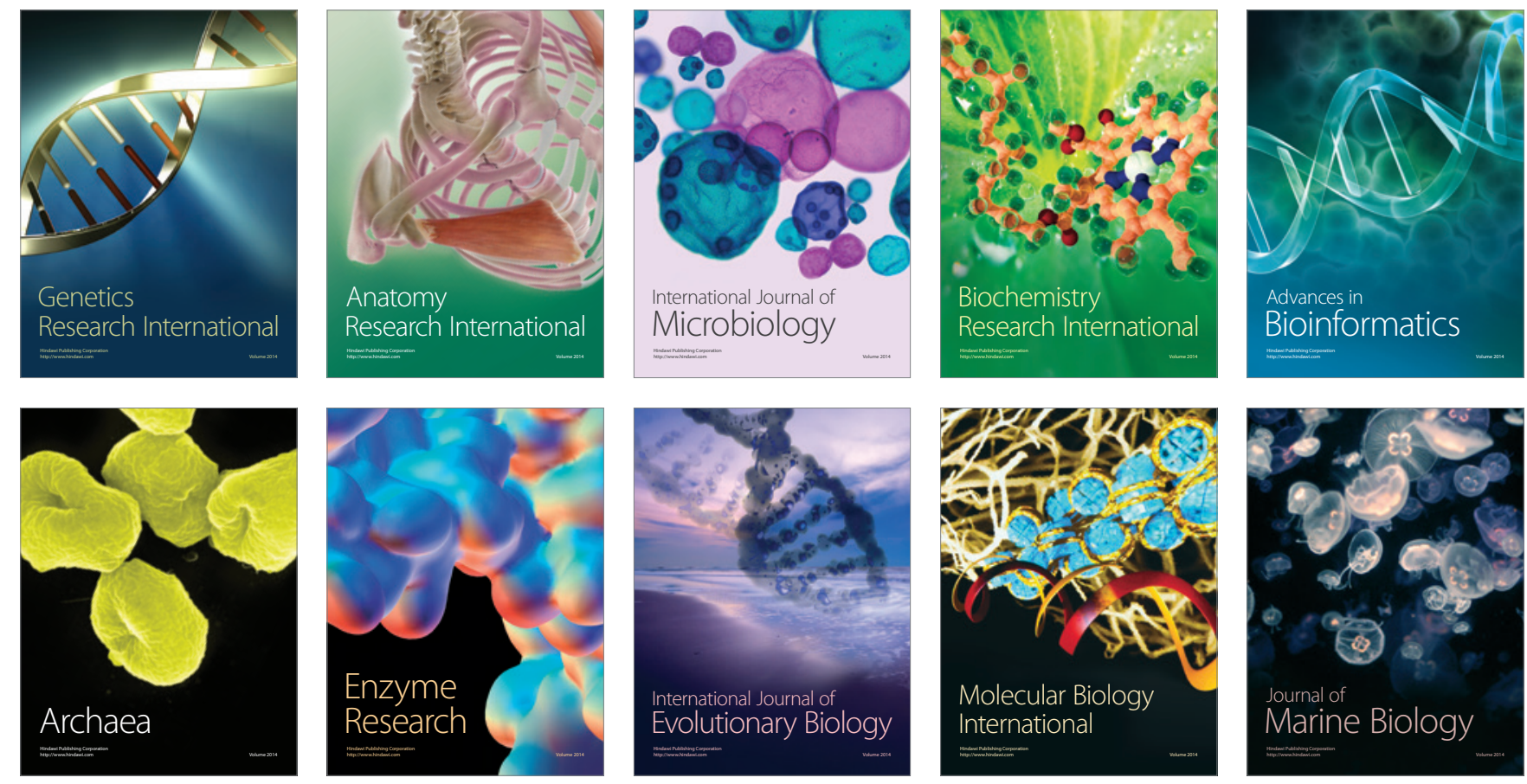